\title{
RADIOCIRURGIA ESTEREOTÃXICA NO TRATAMENTO DE MALFORMAÇÕES ARTERIOVENOSAS CEREBRAIS
}

\author{
JACK BERAHA * \\ CARLOS VITORIO FERIANCIC ** \\ LUIZ ALBERTO MALAGUTI SCAFF ***
}

Tem sido muito difundido o emprego da microcirurgia vascular como a principal indicação no tratamento das malformaçōes arteriovenosas cerebraij (MAV). Em alguns casos, entretanto os riscos inerentes à cirurgia e às seqüelas que dela podem advir fazem com que esta técnica seja desprezada em favor de outras, tais como a embolização terapêtica e as balonizaçoes. Mesmo assim, nas MAV profundas e nas fístulas carótido-cavernosas espontâneas ou tratamentos cirúrgicos, associados ou não a outras técnicas, são muito difíceis e ligados a riscos significativos: hemorragia maciça, déficit de funções cerebrais, falha na obliteração vascular e morte. Nos casos em que as diferentes técnicas são de alto risco ou pouco eficazes, temos como alternativa o método da radiocirurgia estereotáxica (Rdc). A Rdc foi conceituada por Lecksell, em meados de 1951, como sendo todo um procedimento de irradiação localizada que utiliza fontes de energia externa ao crânio, com finalidade terapêutica. É destinada à destruição de pequenos volumes de tecido cerebral, normal ou patológico, de situação geralmente profunda, sendo a localização feita por métodos esterotáxicos 7 . O conceito radiobiológico de que os vasos sangüíneos podem ser obliterados pelas radiações ionizantes, levando a tromboses e hemostasia, fez com que a técnica da Rdc, descrita por vários autores, ficasse cada vez mais em evidência. $O$ estudo morfológico mostra que a parede vascular responde à energia absorvida com proliferação reativa da camada interna, que culmina com a obliteração do lúmen vascular. A aparente diferença entre a reação do tecido endotelial está no calibre dos vasos. Nos pequenos, há estreita margem para alterações endoteliais antes da obliteração. Já nos grandes, apesar da similaridade da alteração, existe distinção no tempo para que ocorra a oclusão completa do lúmen. Fisiopatologicamente, há edema do sub-endotélio vascular com fissuramento das paredes, seguido de hemorragias difusas, formação de trombos, degeneração e necrose das células endoteliais, com aumento dos colóides intersticiais e aumento da atividade fibroblástica. Assim, teriamos a obliteração dos vasos quer pela trombose provocada pelo acréscimo da atividade plaquetária a nível das alterações endoteliais, quer pela obstrução causada pela hialinização e consequente estreitamento dos vasos $8,9,11$.

Constitui o propósito deste trabalho relatar nossa experiência com o emprego da Rdc no tratamento de MAV, baseada na observação de 25 casos estudados no periodo de três anos (1983-1986).

Trabalho realizado no Departamento de Radiocirurgia Estereotáxica do Instituto de Radioterapia Osvaldo Cruz de Sāo Paulo: *Neurocirurgiāo; ** Radioterapeuta; *** Fisico. 


\section{METODOLOGIA}

Sāo descritas técnicas distintas pelos diversos autores, em função do tipo de fontes e sistemas de fixação estereotáxica. Suecos e argentinos utilizam Gamma Unit, em que múltiplas fontes de 60 Co são dispostas em semicírculo adaptando-se a um sistema estereotáxico de Lecksell (7). Os espanhóis usam o telecobalto com uma única fonte móvel (1); os americanos aproveitam o efeito Bragg das partículas pesadas através do Cyclotron (5). No Brasil, a técnica da radiocirurgia, utilizada pioneiramente em nosso Departamento, faz uso de radiação $x$ proveniente de um acelerador linear de $4 \mathrm{MV}(2)$.

Tendo o diagnóstico estabelecido e a indicação da irradiação focalizada, procedemos à localização das MAV intracerebrais por angiografia estereotáxica. Para tal, fixamos quadro estereotáxico na cabeça do paciente, quadro que pertence ao sistema desenvolvido por Barcia-Salorio (um Lecksell modificado) e especialmente adaptado para nosso modus operandi. A angiografia estereotáxica fornece as coordenadas necessárias para a irradiação do alvo em questāo. Temos entāo o tamanho, localizaçāo e magnificação necessários para o procedimento. $O(s)$ alvo(s) pré-determinado(s) é(são) estudado(s) em conjunto com a equipe de física médica, para podermos calcular as curvas de isodose para o(s) alvo(s). O número àe alvos está intimamente ligado ao volume da MAV. Os colimadores utilizados são os de 6, 10 e $15 \mathrm{~mm}$ de diâmetro, que podem ser compostos em combinaçăo ou isolados, com a finalidade de se moldarem à geometria espacial da patologia a ser irradiada. As dosagems para irradiação eståo baseadas nas curvas gráficas de Kjelberg e na vasta experiência dos suecos, que são usadas para nosso sistema $(4,32)$. Procuramos fazer com que a MAV nảo receba menos que 25 Gy na sua periferia, visto ser este valor reconhecidamente o mínimo necessário para ocorrer obliteração da MAV num periodo variável entre um e dois anos de evoluçāo. Feitos estes procedimentos, fixamos o sistema estereotáxico ao isocentro do acelerador linear, que deverá coincidir com o centro do alvo a ser irradiado. Como trabalhamos com uma fonte móvel, quaiquer que seja a posição do cabeçote do acelerador os feixes emitidos passarão pelo centro do sistema. Para que năo ocorram possiveis fatores mecānicos de erro na localização, a irradiação só procede a partir do momento em que todo o sistema estiver verificado, através de feixes de laser instalados lateral e superiormente, coincidindo com o alvo. O cabeçote é deslocado sucessivamente de acordo com os ângulos e portas de entradas pré-selecionados.

Terminada a irradiação o paciente é retirado da aparelhagem e, logo no dia seguinte, terá alta hospitalar. Todo o procedimento é realizado sob anestesia local, salvo nos casos em que o paciente se cncontra agitado. $O$ controle dos pacientes é realizado semestralmente com exames tomográficos e, a partir da obliteração da MAV, procedemos à angiografia cerebral, tendo em conta qua isso ocorre em média. no intervalo de um a dois anos após a irradiação.

\section{CASUfSTICA E RESULTADOS}

No periodo de três anos, 25 casos de MAV foram tratadas por radiocirurgia em nosso serviço:

a. Cinco casos foram irradiados após terem sido submetidos a microcirurgia num primeiro tempo, pelo fato de permanecerem com pequenas massas vasculares malformadas. Destes, quatro apresentavam sequelas cirúrgicas, como hemiplegias que não regrediram mesmo após a irradiação. Obtivemos obliteraçăo completa em dois casos no acompanhamento de dois anos. Os três restantes permanecem em controle.

b. Dois casos em que a MAV estava situada em território da cápsula interna e artérias lenticuloestriadas: obtivemos obliteração embora apresentassem como complicação quadro hemiparético, tendo regredido em aproximadamente 90\% em um dos casos e permanecido inalterado no outro.

c. Um caso apresentou, durante o curso natnral do tratamento, hemorragia no terceiro mês após a irrađiaçăo. Isto não modificou o andamento do seguimento e năo trouxe sequielas para o paciente.

d. Cinco casos apresentavam sindrome convulsiva crônica. Destes, três obtiveram total obliteração; nos outros dois esta foi parcial $\mathbf{( 7 5} \%$ ). Salientamos que nestes 5 casos houve diminuiçăo da freqüência das crises, em um deles havendo remissão total até o momento. 
e. Dois casos, com hemiparesia desde a infância, apresentavam MAV gigantes (5 cm de diâmetro aproximadamente). Após um ano de evolução, submetidos a controles angiográficos, verificou-se nitida reduçăo do volume das MAV, com diminuição da captaçăo do contraste. Clinicamente, observamos melhora nos déficits motores.

f. Dez casos, os restantes, sem alteraçōes clínicas que merecessem destaque: dois apresentam obliteração total; os demais permanecem no periodo de seguimento.
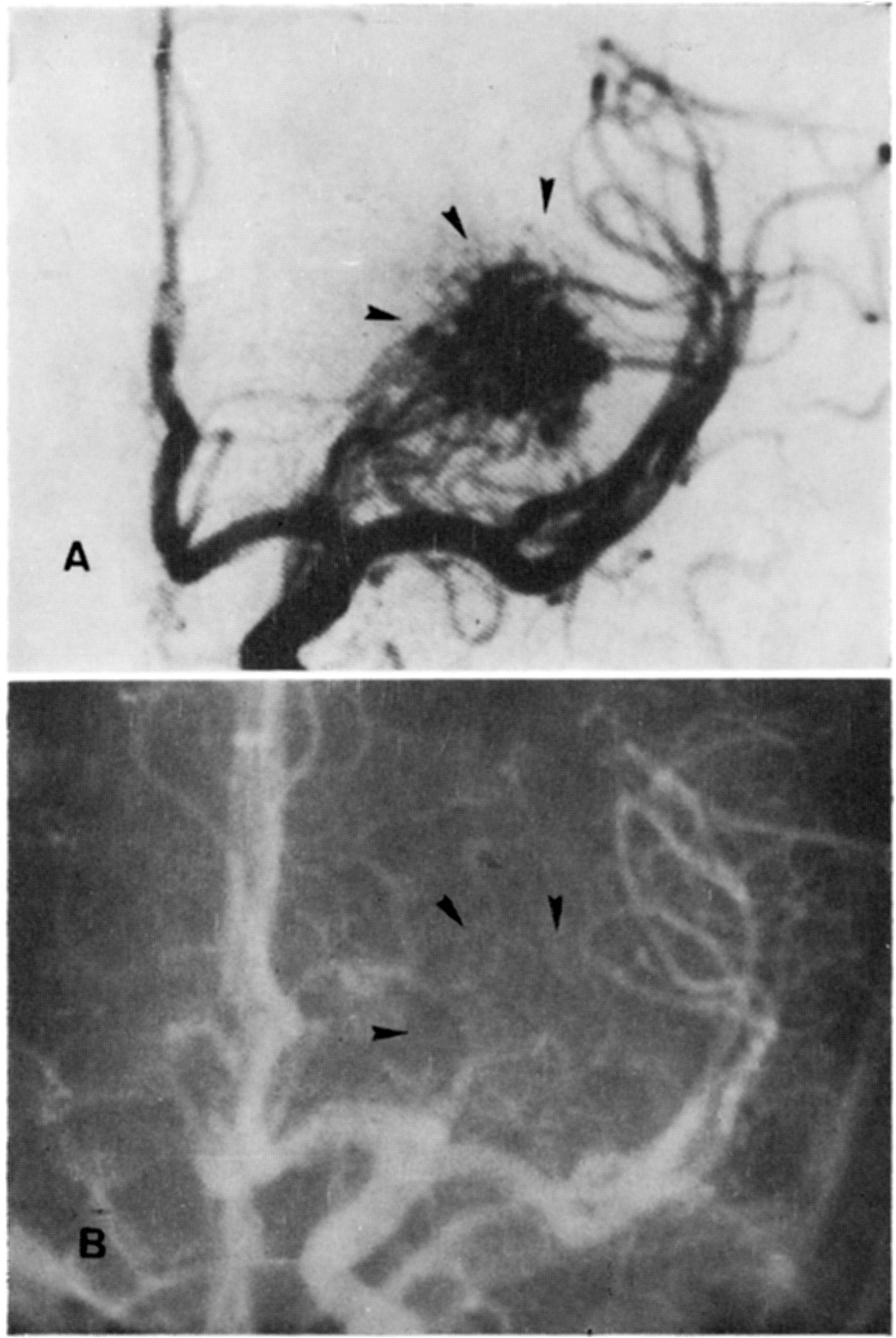

Fig. 1 - Malformação arteriovenosa (antero-posterior): $A$, pré-irradiação; $B$, 18 meses após a irradiação. 
sem que ainda possamos concluir sobre resultados, embora o andamento e as tomografias sejam promissoras.

Assim, em nosso serviço, 25 casos de MAV foram tratados nos últimos três anos. Em nove casos já procedemos aos controles radiológicos, com resultados satisfatórios, como é ilustrado (Figs. 1 e 2). Nos restantes, o acompanhamento mostra boa evolução, o que nos permite ter perspectivas positivas para os próximos exames.
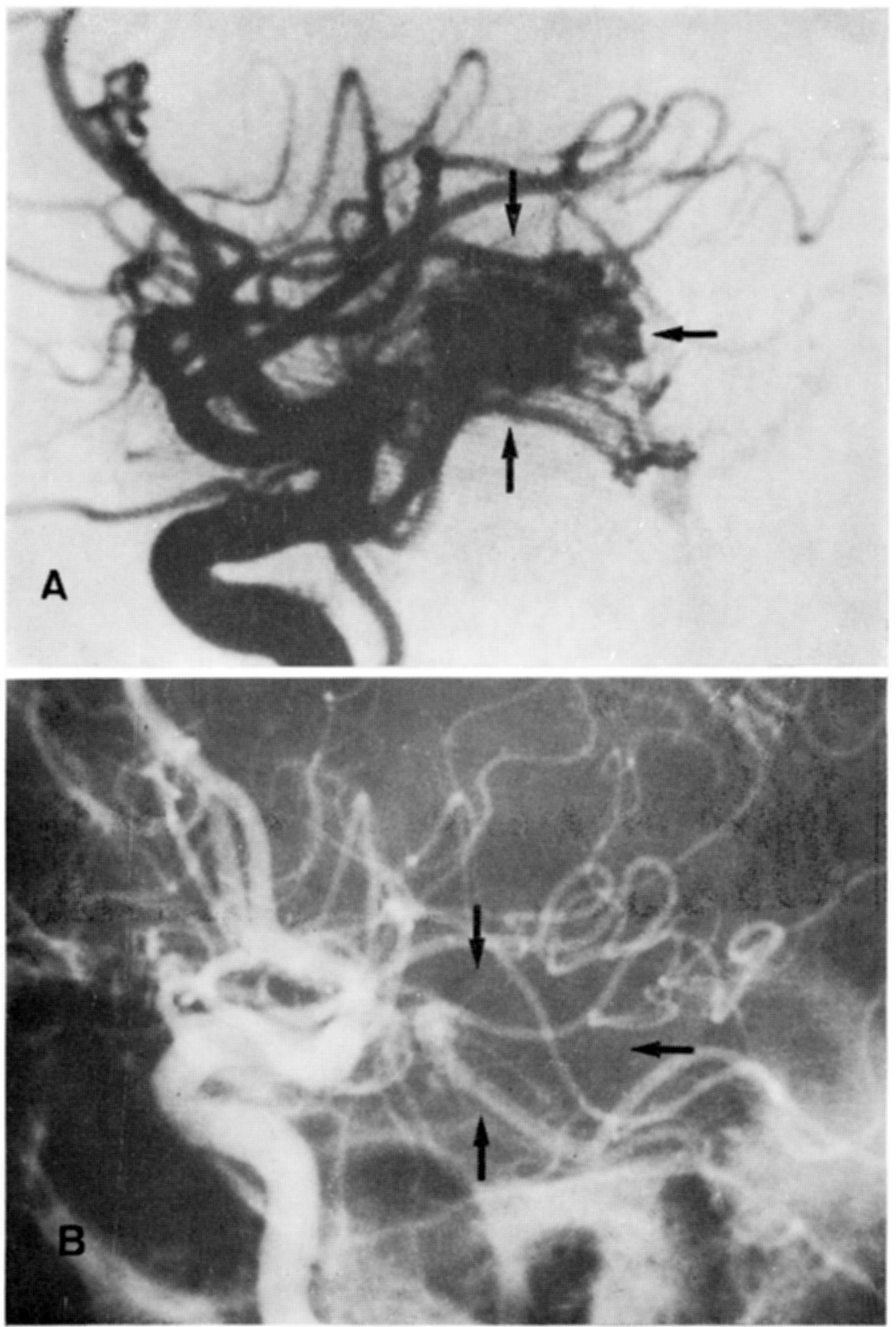

Fig. 2 - Malformação arteriovenosa (perfil): A, pré-irradiação; $B, 18$ meses apbs a irradiação. 


\section{COMENTARIOS}

Uma das dificuldades con que freqüentemente nos deparamos, bem como autores estrangeiros, é quanto à indicação do tratamento radiocirúrgico. Sabe-se que a microcirurgia é o principal método utilizado. Temos procurado irradiar as MAV profundas de difíceis acessos cirúrgicos ou aqueles casos que já tenham sido submetidos a tratamento prévio e nos quais ainda permaneçam resquicios da MAV que não possam ter sido extirpados.

Nos casos em que ocorre mais de um sangramento, a indicação é sempre cirúrgica pois, segundo Olivecrona, $25 \%$ destes pacientes voltam a sangrar em menos de um ano 3,10 . Naqueles em que houve uma única hemorragia ou naqueles em que as MAV foram diagnosticadas na evolução natural de processos como epilepsias, cefaléias e déficits motores, a possibilidade de submetê-los à radiocirurgia é benéfica. $O$ risco de nova hemorragia na história natural destes pacientes, que não se modificam após a irradiação, é de $25 \%$ em 5 anos permitindo, assim, o tempo necessário para a ação dos efeitos radiobiológicos.

Os resultados que vêm sendo obtidos por nós até o presente são semelhantes aos dos autores que já desenvolvem esta técnica há aproximadamente 20 anos 1,6,12. Nos casos de MAV intracerebrais estudados, tratados por radiocirurgia, não houve mortalidade. No momento, a principal seqüela resume-se a um caso de hemiparesia. Na literatura são relatadas alterações edematosas e necroses nos territórios irradiados, com regressão a posteriori. Essas alterações podem, por vezes, ser acompanhadas de sintomatologia clínica 6,12 .

A inocuidade de método, o pequeno risco de hemorragia e o limitado tempo de hospitalização fazem da técnica radiocirúrgica importante coadjuvante no tratamento das MAV. Atualmente é discutivel a irradiação numa fase inicial, com a finalidade de facilitar intervenções cirúrgicas e embolizações num segundo tempo. Portanto, deixamos registrado que a radiocirurgia não pretende ocupar o lugar da cirurgia convencional, mas tornar-se poderoso aliado no tratamento dessas patologias. A boa evolução de nossos casos nos incentiva a continuar a desenvolver a técnica radiocirúrgica, aprimorando esta promissora área complementar da neurocirurgia moderna.

\section{RESUMO}

Neste trabalho os autores pretendem mostrar a utilidade da técnica radiocirúrgica nas malformações arteriovenosas intracerebrais, como procedimento alternativo para estas patologias. São apresentados o procedimento e os resultados verificados em 25 casos tratados durante período de três anos.

\section{SUMMARY}

Stereotaxic radiosurgery for therapy of arteriovenous malformations of the brain.

Since March 1983 we are attempting for the first time in Brazil to develop improvements on stereotactic radiosurgery techniques. We have been using the multifocal irradiation method attaching to this purpose the Barcia IV stereotactic frame to a $4 \mathrm{MV}$ linear accelerator. We show details about the centralisation technique and the security patterns in our system. From October 1983 to November 1986, 25 patients with arteriovenous malformations of the brain were submitted to a radiosurgery treatment in our service. Good results obtained on the follow-up from one to three years are analysed. 


\section{REFERENCIAS}

1. BARCIA-SALORIO, J.L. - Radiocirurgia estereotáctica. Pathos 29:54, 1982.

2. BERAHA, J.; BARCIA-SALoRIO, J.L.; BORDES, J. \& HERNANDEZ, G. Radicirurgia estereotáxica. Arq. bras. Neurocir. 3:15, 1984.

3. FORSTER, D.M.C.; STEINER, L. \& HAKANSON, S. - Arteriovenous malformations of the brain: a long term clinical study. J. Neurosurg. 37:562, 1972.

4. KJELLBERG, R.N. - Bragg peak proton radiosurgery for arteriovenous malformation of the brain. First International Seminar on the use of Proton Beams in Radiation Therapy, Moscow, 1977.

5. KJELLBERG, R.N. - Bragg peak proton radiosurgery method. In G. Szikla: Stereotactic Cerebral Irradiation. Inserm Symposium 12. Elsevier-North Holland, Amsterdam, 1979, pg. 233.

6. KJELLBERG, R.N.; HANAMURA, T.; DAVIS, K.R. \& ADAMS, R.D. - Bragg peak proton beam therapy for arteriovenous malformation of the brain. N. Engl. J. Med. 309:269, 1983.

7. LEKSELL, L. - The stereotaxic method and radiosurgery of the brain. Acta chir. scand. $102: 316,1951$.

8. LEKSELL, L.; LARSSON, B.; ANDERSSON, B.; REXED, B.; SOURANDER, P. \& MAIR, W. - Lesions in depth of the brain produced by a beam of high energy protons. Acta radiol. $54: 252,1951$.

9. NILSSON, A.; WENNERSTRAND, J. \& LEKSELL, L. — Stereotactic gamma irradiation of basilar artery in cat: a pilot study. Acta radiol. oncol. Radioth. phys. biol. $17: 150,1978$.

10. OLIVECRONA, H. \& REEVES, J. - Arteriovenous aneuryms of the brain. Arch. Neurol. 59:567, 1958.

11. SMITH, L.J. - Effects of gamma irradiation on isolated surviving arteries and their vasavasorum. Amer. J. Physiol. 201:901, 1961.

12. STEINER, L. - Treatment of arteriovenous malformations by radiosurgery. In B. Stein: Intracranial Arteriovenous Malformations. Current Neurosurgical Practice, 1984.

Departamento de Radiocirurgia Estereotáxica - Av. Angélica, 2168 - 01928, Säo Paulo, $\$ P-B r a s i l$. 